\title{
RNA-Seq Analysis Reveals Candidate Targets for Curcumin against Tetranychus cinnabarinus
}

\author{
Xuejiao Liu, Dousheng Wu, Yongqiang Zhang, Hong Zhou, Ting Lai, and Wei Ding \\ College of Plant Protection, Southwest University, Chongqing 400716, China \\ Correspondence should be addressed to Wei Ding; dingw@swu.edu.cn
}

Received 18 June 2016; Revised 21 July 2016; Accepted 27 July 2016

Academic Editor: Bernadette F. Ardelli

Copyright (C) 2016 Xuejiao Liu et al. This is an open access article distributed under the Creative Commons Attribution License, which permits unrestricted use, distribution, and reproduction in any medium, provided the original work is properly cited.

\begin{abstract}
Tetranychus cinnabarinus is an important agricultural pest with a broad host range. We previously identified curcumin as a promising acaricidal compound against T. cinnabarinus. However, the acaricidal mechanism of curcumin remains unknown. In this study, RNA-seq was employed to analyze the transcriptome changes in T. cinnabarinus treated with curcumin or the solvent. A total of 105,706,297 clean sequence reads were generated by sequencing, with more than $90 \%$ of the reads successfully mapped to the reference sequence. The RNA-seq identified 111 and 96 differentially expressed genes between curcumin-and solvent-treated mites at 24 and $48 \mathrm{~h}$ after treatment, respectively. GO enrichment analysis of differentially expressed genes showed that the cellular process was the dominant group at both time points. Finally, we screened 23 differentially expressed genes that were functionally identical or similar to the targets of common insecticide/acaricides or genes that were associated with mite detoxification and metabolism. Calmodulin, phospholipase $\mathrm{A}_{2}$, and phospholipase $\mathrm{C}$ were activated upon curcumin treatment suggesting that the calcium channel related genes might play important roles in mite's response to curcumin. Overall our results revealed the global transcriptional changes in T. cinnabarinus after curcumin treatment to enable further identification of the targets of curcumin in mites.
\end{abstract}

\section{Introduction}

The carmine spider mite, Tetranychus cinnabarinus, is a destructive agricultural and forest pest that belongs to class Arachnida, order Acariformes, family Tetranychidae [1]. This mite is widely distributed in warm regions of the world and has an unusually broad host range; the parasite can infect more than 100 plant species from 32 plant families, including beans, cotton, cucumber, tomato, melons, and other horticultural crops or ornamental plants [2-4]. As a phytophagous pest, $T$. cinnabarinus uses the stylet to suck out cell content, thereby causing mechanical damage of the parasitic site and the drying of leaves. Given its small size, short life cycle, high fecundity, and strong adaptability to high temperature, $T$. cinnabarinus is prone to outbreaks during the warm season, thereby causing severe yield and economic losses $[5,6]$.

The control of $T$. cinnabarinus largely relies on the use of insecticides/acaricides. Chemical insecticides/acaricides have long been the dominant intervention because of their quick and efficient acaricidal effect. However, the intensive and unreasonable use of chemical pesticides has recently led to the evolution of resistance in this mite. The short developmental duration and haplodiploid sex determination may accelerate the evolution of pesticide resistance. Studies have shown that the spider mite has a wide record of resistance against almost all acaricidal agents [6-9], which makes this pest the most resistant among arthropods. Consequently, the control of T. cinnabarinus by traditional chemical pesticides became exceedingly challenging. What is worse is that chemical pesticide residues seriously threaten human health and the environment. Therefore, safe and environmentfriendly acaricides should urgently be developed. Botanical acaricides, which have low toxicity to mammals and can be easily degraded, are potential candidates for integrated mite management. Thus far, a few natural products or plant extracts, such as essential oils, terpenoids, alkaloids, and flavonoids, have very promising acaricidal activities against different agricultural mites $[10,11]$.

Our laboratory has focused on the study of botanical acaricides for more than ten years; several plant compounds were 
identified as promising acaricidal compounds [12-15]. As one of the acaricidal compounds we identified, curcumin showed very good repellent activity and oviposition inhibition, as well as high-level acaricidal activity against $T$. cinnabarinus [16]. Interestingly, pyrimidinone, isoxazole, and pyrazole derivatives of curcumin also showed promising acaricidal effects against this mite [17-19]. The underlying biochemical mechanism of curcumin derivatives against T. cinnabarinus was probably the inhibition of the nervous system-related enzyme activity [19]. The acaricidal activity of curcumin has been evaluated, and its possible biochemical mechanism has been investigated. However, the molecular mechanism or molecular targets of curcumin against T. cinnabarinus remain unknown.

Acaricides are transferred once they come into contact with mites; a portion is usually detoxified and excreted by mites, whereas the remaining amount will finally reach the target site to disrupt the normal physiological activities of mites. Based on the type of acaricide, the mechanism of acaricide action is divided into the following categories: interference of the neural signaling process, interference of the insect metabolism and growth processes, inhibition of the activity of certain key enzymes, and the activation or inhibition of some important proteins, including extracellular information-mediated regulation of serine and threonine [20, 21]. The main molecular targets of commonly used pesticides were the gamma-aminobutyric acid (GABA) receptor [22, 23], glutamate-gated chloride channels (GluCls) [5], acetylcholinesterase (AChE) [24], voltage-gated sodium channels [25], cytochrome b [7], and some detoxification enzymes.

RNA-Seq is a robust next generation high-throughput sequencing technology for the detection of gene expression of a species under specific conditions. This technology has recently been widely used for drug target screening and discovery because it provides comprehensive information of gene transcription and gene regulation. To reveal the molecular mechanism and search for candidate molecular targets of curcumin against T. cinnabarinus, we performed RNA-Seq on T. cinnabarinus treated with solvent or curcumin in this study. The obtained reads were mapped to the genome of Tetranychus urticae [26], a sister species of T. cinnabarinus [27]. The gene expression levels were calculated and compared to identify differentially expressed genes in solvent- or curcumintreated $T$. cinnabarinus. The annotated functions of the differentially expressed genes were further analyzed. Finally, we confirmed our RNA-Seq data by quantitative real-time PCR.

\section{Materials and Methods}

2.1. Mite Rearing. A colony of T. cinnabarinus was originally collected from cowpea leaves growing in a field in Beibei, Chongqing, China; this colony had been maintained in the lab for at least 16 years without exposure to any pesticides [12]. T. cinnabarinus was reared on cowpea seedlings (Vigna unguiculata) in an artificial climate chamber at $26 \pm 1^{\circ} \mathrm{C}$ and $60 \%-80 \%$ relative humidity, with a $14 \mathrm{~h}$ light/10 h dark photoperiod. Prior to the experiment, a few 3-5-day-old female adults were transferred onto mite-free cowpea seedlings to spawn for $6 \mathrm{~h}$ before the female adults were removed. After incubation in the climate chamber for 7 days, new female adults were obtained from the inoculated seedlings and used for downstream acaricide treatment.

2.2. Curcumin Treatment. Curcumin was dissolved in sterile distilled water containing $0.25 \%$ Tween 80 and $3 \%$ acetone to a final concentration of $2.64 \mathrm{mg} / \mathrm{mL}$, the median lethal concentration $\left(\mathrm{LC}_{50}\right)$ of curcumin against $T$. cinnabarinus. For curcumin treatment, more than 300 female adults were transferred onto three fresh potted cowpea leaves which were placed in a small Petri dish containing some water. The leaves were sprayed with the curcumin solution with the abovementioned concentration. Sterile distilled water with $0.25 \%$ Tween 80 and 3\% acetone was used as the solvent control. Three Petri dishes from one independent experiment comprised a replicate and two biological replicates were used for RNA purification and library preparation. All treated mites were maintained at $26 \pm 1{ }^{\circ} \mathrm{C}$ and $60 \%-80 \%$ relative humidity under a $14 \mathrm{~h}$ light $/ 10 \mathrm{~h}$ dark cycle. At $24 \mathrm{~h}$ posttreatment and $48 \mathrm{~h}$ posttreatment, all the live mites from each treatment were collected, immediately frozen in liquid nitrogen, and stored in a $-80^{\circ} \mathrm{C}$ freezer for RNA extraction.

\subsection{RNA Extraction, Library Preparation, and Sequencing.} Total RNA of each sample was extracted with the RNeasy ${ }^{\circledR}$ plus Micro Kit (Tiangen, Beijing, China) according to the manufacturer's instructions. RNase-free DNase I (Tiangen, Beijing, China) was used to remove any genomic DNA contamination. The quality of RNA was examined in terms of several aspects. First, 1\% agarose gels were used to monitor RNA contamination and degradation. The RNA purity was checked by NanoDrop ${ }^{\mathrm{TM}}$. Finally, the RNA concentration, RIN, and 28S/18S were determined with the RNA Nano 6000 Assay Kit of the Agilent Bioanalyzer 2100 system (Agilent Technologies, CA, USA).

The polyA mRNA was enriched from the total RNA with the oligo(dT) magnetic beads (Dynabeads mRNA Purification Kit, Invitrogen) and digested into short fragments ( $130 \mathrm{bp}$ ) with First-Strand Buffer (Invitrogen) under elevated temperature. The first-strand cDNA was synthesized with random hexamer primers, the First-Strand Master Mix, and Super Script II reverse transcriptase (Invitrogen). The second-strand CDNA was subsequently synthesized with the Second-Strand Master Mix. After adenylation of $3^{\prime}$ of ends of DNA fragments, the sequencing adaptors were ligated. The synthesized cDNA were purified with AMPure XP beads to select fragments with an average length of $130 \mathrm{bp}$; these cDNA were eluted in EB buffer, followed by PCR amplification. The quality of the library and the concentration of cDNA were checked with the Agilent 2100 Bioanalyzer. The prepared libraries were sequenced on the Ion Proton platform (BGI, Shenzhen, China) with the sequencing strategy of single-end $150 \mathrm{bp}$.

\subsection{Processing and Mapping of RNA-Seq Data. Primary} sequencing data was produced by Ion Proton and called raw reads. These data were first subjected to quality control. The raw reads were filtered by discarding low-quality sequences 
TABLE 1: Statistics of the reads generated from the RNA-Seq and their mapping against T. urticae genome.

\begin{tabular}{lcccc}
\hline Sample $^{\mathrm{a}}$ & Total reads & Total base pairs & Total mapped reads & Percentage of mapping \\
\hline CK 24 h-1 & 13083313 & 1838765016 & 12714468 & $97.18 \%$ \\
CK 24 h-2 & 13583410 & 1915413262 & 12943362 & $95.29 \%$ \\
CK 48 h-1 & 12748755 & 1810155299 & 11990145 & $94.05 \%$ \\
CK 48 h-2 & 12871381 & 1812264301 & 12127836 & $94.22 \%$ \\
Curcumin 24h-1 & 13031623 & 1847855454 & 12652036 & $97.09 \%$ \\
Curcumin 24h-2 & 13499723 & 1966705882 & 12674097 & $93.88 \%$ \\
Curcumin 48 h-1 & 13297084 & 1758353497 & 12559389 & $94.45 \%$ \\
Curcumin 48 h-2 & 13591008 & 1768841760 & 13020334 & $95.80 \%$ \\
\hline
\end{tabular}

${ }^{\mathrm{a}}$ Two replicates for each treatment at each time point.

and removing adaptor sequences. The Q20, Q30, and GC content of the filtered reads were calculated and checked. All the obtained high-quality and clean reads were mapped against the reference genome of $T$. urticae with T-Map (Version 3.4.1, parameter mapall -a 2 -n $8-\mathrm{v}-\mathrm{Y}-\mathrm{u}$-o 1 stage 1 map 4) (http://www.tamariskmap.org/cwis438/websites/tmap/home.php?WebSiteID=2). Mismatches of three or less than three bases per read (average length $=150 \mathrm{bp}$ ) were allowed in the mapping. Both unique and nonunique mapped reads were used for mapping percentage calculation. The read per kilobase per million mapped reads (RPKM) of each gene was calculated by the following formula: RPKM = total exon reads/mapped reads in million $X$ exon lengths in $\mathrm{kb}$. This value was used as expression level for differential expression analysis.

2.5. Differential Expression Analysis. To identify differentially expressed genes between different treatments, a rigorous algorithm was used as previously described [28]. The false discovery rate (FDR) was calculated to determine the threshold $p$ value in multiple tests. In the present study, the threshold was FDR $\leq 0.001$, with an absolute value of $\log _{2}$ ratio $\geq 1$, to determine the significance of differences in gene expression [29]. For in-depth analysis of differentially expressed genes, we first performed cluster analysis with Cluster software and Java TreeView software. Finally, we mapped all the differentially expressed genes to terms in the KEGG and GO databases for gene annotation.

2.6. Quantitative Real-Time PCR Analysis. The differential expression of some genes generated by the abovementioned parameters was validated by quantitative real-time PCR (qRT-PCR). Fifteen genes were randomly chosen from significantly differentially expressed genes and used for qRTPCR confirmation. All the primers used in this study were designed by Primer 3.0 (http://frodo.wi.mit.edu/) and are listed in Table S1 in Supplementary Material available online at http://dx.doi.org/10.1155/2016/2796260. RPS18 was used as the reference gene for gene expression correction [30]. qRTPCR analyses were performed on the CFX96 Manager (Bio$\mathrm{Rad}$ ) in a $20 \mu \mathrm{L}$ reaction, which included $10 \mu \mathrm{L}$ of Sso Fast TM EvaGreen ${ }^{\circledR}$ Supermix (Bio-Rad), $0.2 \mathrm{mM}$ of each of the forward and reverse primers, $1 \mu \mathrm{L}$ of 1 to 10 diluted cDNA, and $7 \mu \mathrm{L}$ of MilliQ $\mathrm{H}_{2} \mathrm{O}$. The PCR amplification profile was as follows: $95^{\circ} \mathrm{C}$ for $5 \mathrm{~min}$, followed by 40 cycles of $95^{\circ} \mathrm{C}$ for $10 \mathrm{~s}$ and $60^{\circ} \mathrm{C}$ for $20 \mathrm{~s}$. Melting curves from $60^{\circ} \mathrm{C}$ to $95^{\circ} \mathrm{C}$ were run to determine the consistency and specificity of the products. The quantification of expression levels was analyzed by the $\Delta \Delta$ Cq method [31].

\section{Results}

3.1. RNA-Seq Data Analysis. To investigate the transcriptional changes in T. cinnabarinus after curcumin treatment, the purified mRNA from curcumin- or solvent-treated mites were sequenced on the Proton platform. After removal of duplicate sequences, adaptor sequences, and low-quality reads, a total of $53,419,438$ clean sequence reads were generated from curcumin-treated mites and 52,286,859 clean sequence reads were generated from solvent-treated mites (Table 1). These clean reads were used for further mapping and processing. All the sequencing data have been submitted to the GEO web site (http://www.ncbi.nlm.nih.gov/geo/) with the accession number GSE80001. Given that the whole genome sequence of $T$. cinnabarinus is still unavailable, the genome information of T. urticae was used as the reference genome for mapping reads. More than $90 \%$ of these clean reads could be successfully mapped to the reference genome, thereby suggesting the close genetic relationship between $T$. cinnabarinus and T. urticae, as well as the overall good quality of RNA-Seq. Sequencing saturation analysis showed that the number of identified genes almost reached saturation when the total read numbers approached 5 million per sample (Figure S1). Each of our libraries generated more than 10 million reads, thereby indicating that the sequencing depth is sufficient to cover most of the transcripts in this organism.

3.2. Differential Gene Expression between Curcumin- and Solvent-Treated Mites. There are a total of 18,414 proteincoding gene models in the T. urticae genome database [26]. By mapping all the clean reads to the reference genome, we found that more than 14,000 genes were detected for expression in each sample (Table S2). Gene expression level was calculated based on the RPKM value, which integrates the effect of gene length on read number and sequencing depth. With a cutoff of FDR $\leq 0.001$ and fold change $\geq 2$, 111 and 96 genes were identified as significantly differentially expressed genes between curcumin- and solvent-treated 


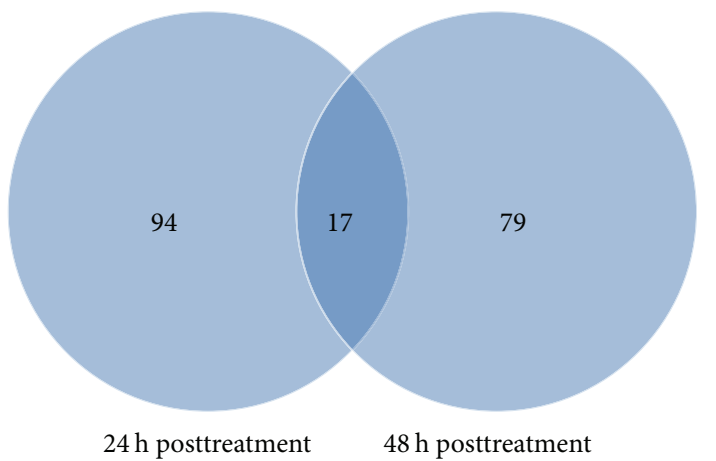

(a)

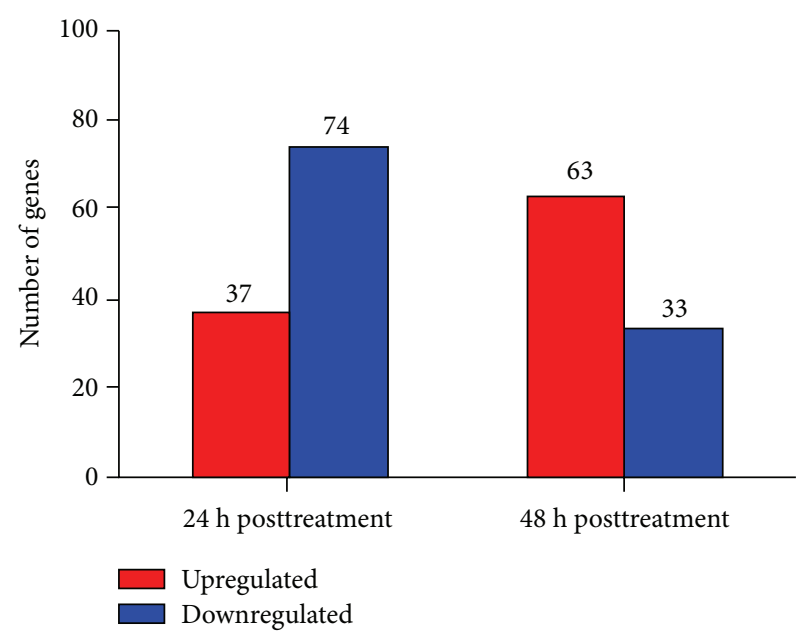

(b)

Figure 1: Distribution of differentially expressed genes in T. cinnabarinus in response to curcumin. (a) Venn diagram showing the total number of differentially expressed genes at $24 \mathrm{~h}$ and $48 \mathrm{~h}$ posttreatment and the number of overlapped genes between two time points. (b) The number of upregulated and downregulated genes at $24 \mathrm{~h}$ and $48 \mathrm{~h}$ after curcumin treatment.

mites at $24 \mathrm{~h}$ and $48 \mathrm{~h}$ posttreatment, respectively (Tables S3 and S4). We compared the differentially expressed genes at two time points and found that most genes were time pointspecific, except for 17 genes that were shared by two time points (Figure 1(a) and Table S5). Among the differentially expressed genes, 37 genes were upregulated and 74 genes were downregulated by curcumin at $24 \mathrm{~h}$ posttreatment, whereas 63 genes were upregulated and 33 genes were downregulated by curcumin at $48 \mathrm{~h}$ posttreatment (Figure $1(\mathrm{~b})$ ). The $\log _{2}$ fold change was from -12.4 to 15.5 . At $24 \mathrm{~h}$ posttreatment, the number of downregulated genes was twice the number of upregulated genes. At $48 \mathrm{~h}$ posttreatment, more genes were upregulated by curcumin. This difference suggests that the overall transcriptional level in mites changed with the increase of curcumin treatment time.

3.3. GO Enrichment and KEGG Pathway Analysis of Differentially Expressed Genes. GO database is a widely used database for annotating sequences, genes, and gene products. To better understand the molecular function of genes involved in the response of $T$. cinnabarinus to curcumin treatment, all the differentially expressed genes were mapped to terms in GO database and compared with the whole reference genome background (Tables S6 and S7). The mapping results showed that differentially expressed genes from $24 \mathrm{~h}$ posttreatment were categorized into $35 \mathrm{GO}$ subgroups (Figure 2(a)) and differentially expressed genes from $48 \mathrm{~h}$ posttreatment were categorized into $25 \mathrm{GO}$ subgroups (Figure 2(b)). For both time points, more genes were categorized into biological process as compared with the number of genes that were assigned to molecular function and cellular component. The distribution of the GO subgroup indicated that cellular process $(80.77 \%)$ under the biological process category was the most represented subgroup throughout the GO classification. The single-organism (65.38\%) and metabolic process $(50 \%)$ subgroups were also highly enriched in the biological process at both time points. At $24 \mathrm{~h}$ posttreatment, one enriched term (DNA topoisomerase activity) had a proportion of $12.5 \%$. At $48 \mathrm{~h}$ posttreatment, three enriched terms were calcium channel complex $(33.3 \%)$, regulation of ion transmembrane transport (25\%), and regulation of transmembrane transport (25\%). In the cellular component category, the subgroups of cell $(83.3 \%)$ and cell part $(83.3 \%)$ were dominant. A larger proportion of genes that were identified in the category of molecular function were involved in catalytic activity, binding, and transporter activity. Interestingly, the most categorized subgroups were relatively similar for 24 and $48 \mathrm{~h}$ posttreatment, thereby indicating a similar response pattern of mites towards curcumin treatment at different time points.

For further characterization of the differentially expressed genes, we performed pathway enrichment analysis with KEGG, a major pathway-related database (Tables S8 and S9). This analysis identified the top 20 enriched pathways for both time points (Figure 3). Protein processing in endoplasmic reticulum was the most represented biochemical pathway among the top 20 pathways at 24 and $48 \mathrm{~h}$ posttreatment. Pathways like MAPK signaling pathway, Huntington's disease, and focal adhesion were also significantly enriched at $24 \mathrm{~h}$ posttreatment (Figure 3(a)), whereas phosphatidylinositol signaling system, salivary secretion, and calcium signaling pathway were well represented at $48 \mathrm{~h}$ posttreatment (Figure 3(b)).

\subsection{Identification of Candidate Genes Involved in Mite Detox-} ification and Insecticide Metabolism. Based on our previous analysis on differentially expressed genes, the candidate genes that associated with mite detoxification and insecticide metabolism (e.g., cell proliferation, cell apoptosis, substance transportation, metabolism, and detoxification) were manually selected (Tables 2 and 3 ). The gene products of these candidates could be classified into several categories, such as signal transduction protein, apoptosis 


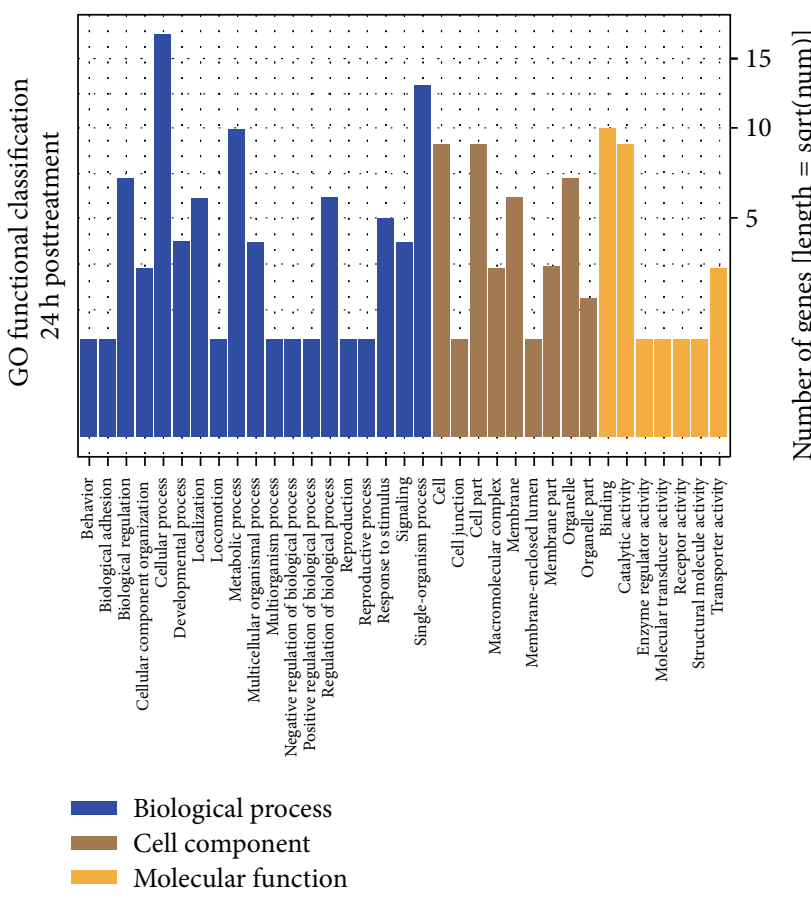

(a)

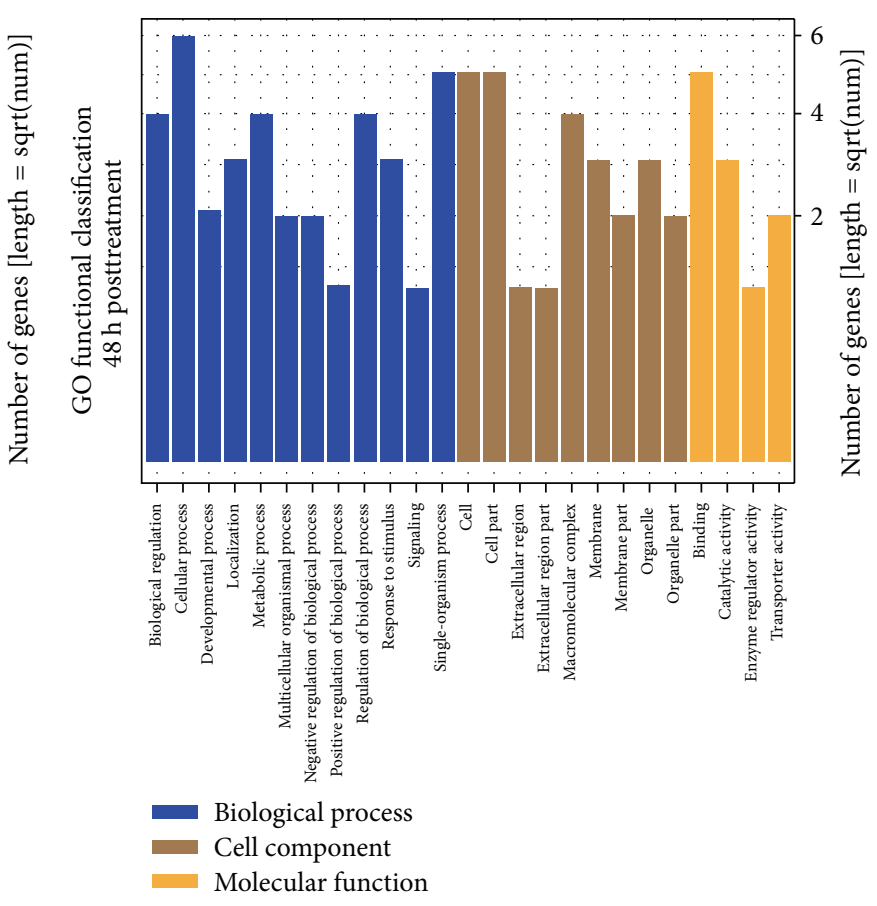

(b)

FIGURE 2: Gene Ontology (GO) enrichment analysis of differentially expressed genes in T. cinnabarinus after curcumin or solvent treatment. (a) Functional categories of differentially expressed genes at $24 \mathrm{~h}$ posttreatment. (b) Functional categories of differentially expressed genes at $48 \mathrm{~h}$ posttreatment. Three main categories, biological process, cellular component, and molecular function, are summarized.

TABLE 2: Selected genes involved in mite detoxification and insecticide metabolism at $24 \mathrm{~h}$ posttreatment.

\begin{tabular}{|c|c|c|c|c|}
\hline \multirow{2}{*}{ Gene ID } & \multirow{2}{*}{ Description } & \multicolumn{2}{|c|}{ RPKM } & \multirow{2}{*}{$\log _{2}$ fold change } \\
\hline & & CK & Curcumin & \\
\hline tetur19g01690 & $\mathrm{ABC}$ transporter & 11.1858 & 2.50684 & -2.16 \\
\hline tetur10g02970 & Topoisomerase & 15.93595 & 5.19572 & -1.62 \\
\hline tetur25g00970 & Hyperpolarization-activated ion channel & 18.6278 & 7.948185 & -1.23 \\
\hline tetur04g07610 & RabGAP/TBC & 15.34715 & 6.826305 & -1.17 \\
\hline teturl0g04980 & Ser/Thr protein kinase & 62.09965 & 27.79795 & -1.16 \\
\hline tetur05g08080 & Similar to gamma-aminobutyric acid & 15.4435 & 39.44995 & 1.35 \\
\hline tetur09g03650 & Cystatin & 308.967 & 845.5195 & 1.45 \\
\hline tetur39g00730 & Vitellogenin 1 & 1.80895 & 7.934175 & 2.13 \\
\hline tetur23g01300 & Glycerol-3-phosphate dehydrogenase & 3.69663 & 17.1982 & 2.22 \\
\hline tetur08g03710 & Ras GTPase & 1.07431 & 7.073055 & 2.72 \\
\hline tetur05g07400 & Phospholipase $\mathrm{A}_{2}$ & 0.253237 & 4.52485 & 4.16 \\
\hline tetur453g00010 & Neuromedin-K receptor & 0.01 & 4.774815 & 8.90 \\
\hline tetur02g05380 & Scaffold protein & 0.01 & 5.95338 & 9.22 \\
\hline
\end{tabular}

protein, detoxified protein, and channel protein, according to their biological functions. Genes involved in signal transduction were Ser/Thr protein kinase, gamma-aminobutyric acid, neuromedin-K receptor, neuropeptide precursor, a phospholipase $\mathrm{C}$ family member, and calmodulin. We also identified topoisomerase, calnexin, RabGAP/TBC, and Ras GTPase as cell apoptosis-related products. In addition, a detoxification-related $\mathrm{ABC}$ transporter protein was detected. Among these selected candidate genes, signal transductionassociated genes were dominant. Interestingly, some candidates, such as Ras GTPase, neuropeptide precursor, and gamma-aminobutyric acid, were upregulated at $24 \mathrm{~h}$ posttreatment but downregulated at $48 \mathrm{~h}$ posttreatment.

Some insecticide targets that have been described for commonly used insecticides were identified in our curcumintreated T. cinnabarinus transcriptome profile (Tables 2 and $3)$. These genes included the ABC transporter and gammaaminobutyric acid. However, except for its acaricidal activity, curcumin is a widely used medicine for human beings. Surprisingly, genes that have similar functional annotations to the targets of curcumin were also found in our study. For example, guanylate kinase, Ras GTPase, and Ser/Thr protein 
TABLE 3: Selected genes involved in mite signal transmission and insecticide metabolism at $48 \mathrm{~h}$ posttreatment.

\begin{tabular}{lcccc}
\hline Gene ID & Description & RK & Curcumin & Log fold change \\
\hline tetur08g03710 & Ras GTPase & 6.75555 & 0.01 & -9.40 \\
tetur07g05920 & Guanylate kinase & 71.2255 & 2.55663 & -4.80 \\
tetur39g00730 & Vitellogenin 1 & 17.40335 & 2.688785 & -2.69 \\
tetur22g02010 & SIFa: neuropeptide precursor & 15.8693 & 2.6419165 & -2.59 \\
tetur05g08080 & Similar to gamma-aminobutyric acid & 23.43175 & 5.391865 & -2.12 \\
tetur28g01480 & Stress-associated reticulum protein 1 & 402.0815 & 183.792 & -1.13 \\
tetur61lg00020 & Calnexin & 17.2701 & 39.63885 & 1.20 \\
tetur05g03410 & Phospholipase C family member & 6.61663 & 18.364 & 1.47 \\
tetur09g03650 & Cystatin & 172.78 & 610.0945 & 1.82 \\
tetur14g03800 & Similar to PMP1 protein & 1.9205 & 10.75305 & 2.49 \\
tetur04g01900 & Calmodulin & 0.0771045 & 9.01032 & 6.87 \\
tetur01g05810 & Exostosin-1 & 0.01 & 39.373 & 11.94 \\
tetur04g07080 & $\alpha$-D-Phosphohexomutase & 0.01 & 141.539 & 13.79 \\
\hline
\end{tabular}

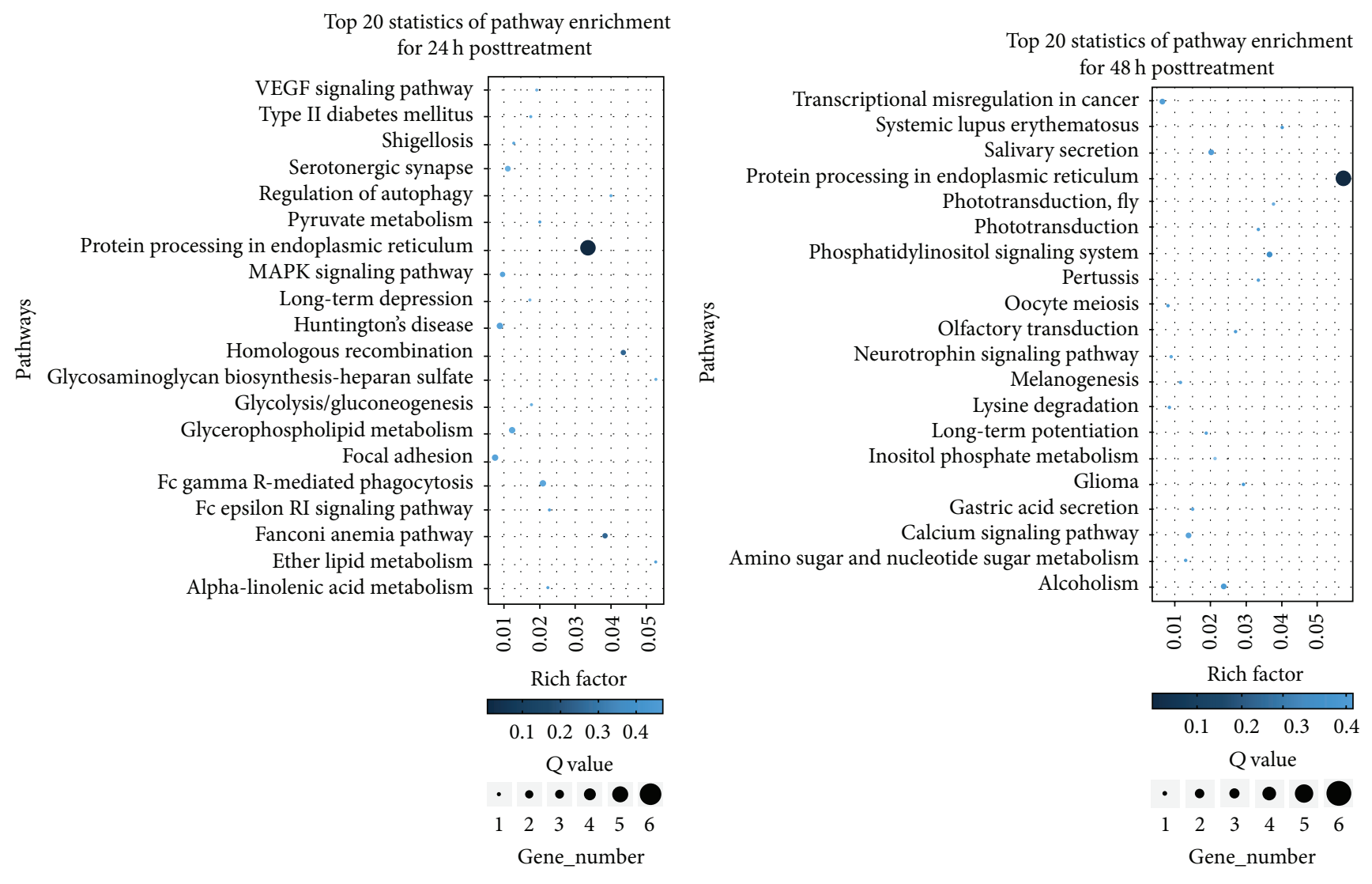

(a)

(b)

FIGURE 3: KEGG pathway analysis of differentially expressed genes in T. cinnabarinus in response to curcumin. (a) Top 20 enriched KEGG pathways of differentially expressed genes at $24 \mathrm{~h}$ posttreatment. (b) Top 20 enriched KEGG pathways of differentially expressed genes at $48 \mathrm{~h}$ posttreatment. Rich factor is defined by the ratio of the number of differentially expressed genes enriched in the pathway and the number of all genes enriched in the same pathway.

kinase were differentially expressed in mites treated with curcumin.

3.5. Validation of RNA-Seq Data by qRT-PCR. To confirm the RNA-Seq results, ten downregulated genes and five upregulated genes were selected from the differentially expressed genes for qRT-PCR analysis. Seven of those selected genes are involved in mite detoxification and insecticide metabolism either at $24 \mathrm{~h}$ or at $48 \mathrm{~h}$ posttreatment. The qRT-PCR experiment showed that all the tested genes had 


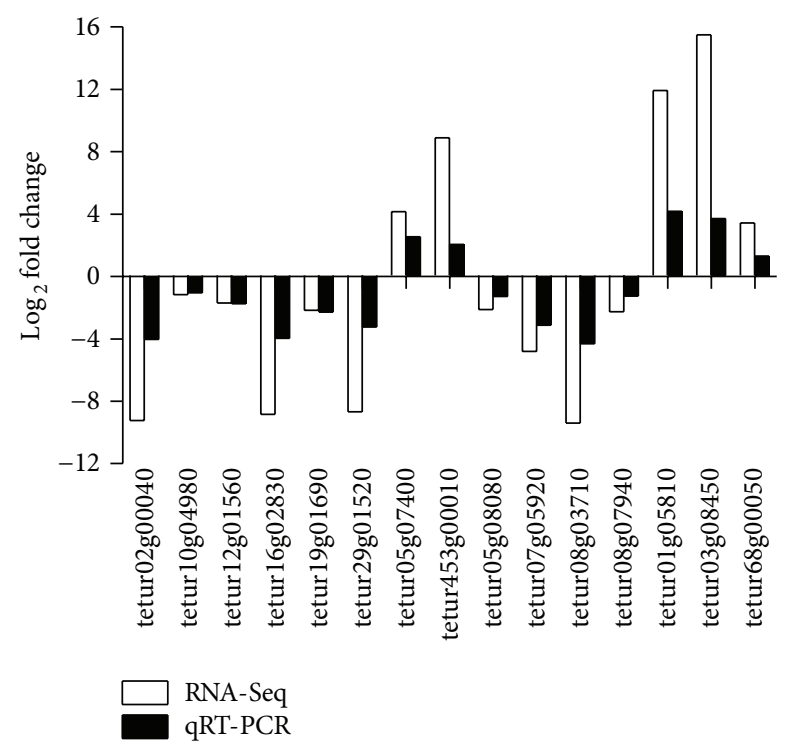

FIGURE 4: qRT-PCR validation of some differentially expressed genes in RNA-Seq. The relative expression levels of fifteen differentially expressed genes in curcumin- or solvent-treated $T$. cinnabarinus were determined by qRT-PCR. RPS18 was used as the reference gene to normalize the gene expression using the $\Delta \Delta \mathrm{Cq}$ method. The $y$-axis indicates the $\log _{2}$ fold change of each gene in qRT-PCR (black) and RNA-Seq (white).

a similar differential expression trend as compared with the RNA-Seq data (Figure 4). For example, the phospholipase $\mathrm{A}_{2}$ gene tetur05g07400 and the neuromedin-K receptor tetur453g00010 were upregulated by a 4.2 and $8.9 \log _{2}$ fold change, respectively, in the RNA-Seq; they were upregulated by a 2.6 and $2.1 \log _{2}$ fold change, respectively, in qRTPCR. The $\log _{2}$ fold change of some differentially expressed genes in the qRT-PCR did not perfectly match that in the RNA-Seq, which was probably attributed to the calculation and sequencing biases. Overall, the qRT-PCR results almost validated the direction of upregulation and downregulation obtained from the RNA-Seq results.

\section{Discussion}

As a botanical compound extracted from Curcuma longa, curcumin has various pharmacological activities, especially strong anticancer activity. Curcumin can affect and disrupt the proliferation, growth, and metabolism of tumor cells, as well as promote apoptosis $[32,33]$. The inhibition of ornithine decarboxylase, $c-m y c$, Akt, and protein kinase $\mathrm{C}$ is considered the major molecular mechanism of curcumin as a pharmaceutical [34-38]. However, the potential mechanism of curcumin as a plant-derived acaricide against T. cinnabarinus is still unclear. In the present study, we applied transcriptomics on $T$. cinnabarinus treated with curcumin or the solvent. Interestingly, we found some differentially expressed genes (e.g., gamma-aminobutyric acid, calmodulin, phospholipase family members, and neuropeptides) that are functionally identical or similar to the targets of common acaricides or that are involved in mite detoxification and metabolism. In addition, we also found some differentially expressed genes that are associated with signal transduction (e.g., guanylate kinase and Ser/Thr protein kinase) and cell apoptosis (e.g., Ras GTPase) which are related to the targets of curcumin as a drug.

A total of 53,198,069 and 52,508,228 reads were obtained from $T$. cinnabarinus treated with curcumin or solvent at 24 and 48 h posttreatment, respectively (Table 1). Given that T. cinnabarinus and T. urticae Koch are sister species, the genome sequence of $T$. urticae Koch was used as reference for mapping. More than $90 \%$ of the reads were successfully mapped to the reference genome, thereby providing good mapping results for downstream analysis. We identified 111 and 96 differentially expressed genes upon curcumin treatment at 24 and $48 \mathrm{~h}$ posttreatment, respectively. The number of downregulated genes was much higher than that of upregulated genes at $24 \mathrm{~h}$ posttreatment (Figure 1(b)), thereby suggesting that more genes were inhibited by curcumin at this time point. However, contradictory results were obtained at $48 \mathrm{~h}$ posttreatment. In addition, the fold changes of some differentially expressed genes like Ras GTPase, gammaaminobutyric acid, and vitellogenin 1 are also different between two time points. The differential expression here is closely related to a previous report that the poisoning symptoms of T. cinnabarinus after curcumin treatment change over time [39]. These symptoms also indicate that different lethal or defense responses might be involved in T. cinnabarinus treated with curcumin at different time points.

Among the differentially expressed genes identified in our study, calmodulin (CaM) and phospholipase $\mathrm{C}$ family members were upregulated in $T$. cinnabarinus upon curcumin treatment (Tables 2 and 3). As the receptor protein of $\mathrm{Ca}^{2+}$, CaM has an important regulatory effect on $\mathrm{Ca}^{2+}$ dependent cell functions and enzyme systems. The $\mathrm{Ca}^{2+} \cdot \mathrm{CaM}$ complex is formed when $\mathrm{CaM}$ is activated. The $\mathrm{Ca}^{2+} \cdot \mathrm{CaM}$ complex targets enzymes such as phosphodiesterase and protein kinase and leads to the conformational change of target enzymes, thereby regulating apoptosis, muscle contraction, intracellular movement, nerve growth, and immune response. In addition, calmodulin can increase the activity of phospholipase [40]. The activation of phospholipase causes the overload of $\mathrm{Ca}^{2+}$ and the excessive activation of calmodulin signaling pathway, thereby resulting in nerve cell death [41]. The activation of CaM in our study might increase the $\mathrm{Ca}^{2+}$ concentration inside cells and trigger nerve cell death, which further causes the death of mites. In addition, the upregulation of phospholipase $\mathrm{A}_{2}$ by curcumin was also found in this study. The activity of phospholipase $A_{2}$ has a vital influence on the intracellular $\mathrm{Ca}^{2+}$ concentration. Overexpression of phospholipase $\mathrm{A}_{2}$ can lead to hydrolysis of membrane phospholipids, as well as an increase in the arachidonic acid release and the production of a large amount of free radicals, which disrupt the calcium balance and cause calcium-dependent cell death $[42,43]$. On the other hand, some signal transduction-related genes, such as the neuromedin-K receptor, and some fat metabolism-associated genes, like glycerol-3-phosphate dehydrogenase [44], are upregulated in phospholipase $\mathrm{A}_{2}$-overexpressing mites. This trend suggests that the overexpression of phospholipase $A_{2}$ 
is likely to open the calcium channels and boost signal transduction, thereby causing excessive excitability of postsynaptic neurons and constant twitching of mites until energy exhaustion and death.

Aside from some upregulated genes, we also identified some genes that were inhibited by curcumin, such as the $A B C$ transporter. Except for cytochrome P450 monooxygenase, carboxylesterase, and glutathione-S-transferase, the $\mathrm{ABC}$ transporter protein is another detoxification protein. The $\mathrm{ABC}$ transporter is linked to the transport and resistance mediated by 27 pesticides, which belong to 9 different categories (e.g., carbamates, neonicotinoids, organophosphates, and pyrethroids) $[45,46]$. As an external drainage pump, its increased gene expression can reduce the concentration of exogenous substances inside cells; otherwise, pesticide accumulation in insects is promoted and the detoxification and metabolism in mites are disrupted, thereby accelerating the death of mites [47]. Meanwhile, the downregulation of $\mathrm{ABC}$ transporters could inhibit the growth of insects and lead to abnormal formation of the stratum corneum, as well as spawning and hatching defects, thereby significantly increasing mortality [48]. In our case, the $\mathrm{ABC}$ transporter gene was inhibited by curcumin, which suggests that the detoxification function is disturbed in curcumin-treated mites to promote the death of these insects. However, this was not the case in a previous study in which $\mathrm{ABC}$ transporter was activated upon treatment with plant-derived compound $\beta$-sitosterol [49]. This is probably because the mite strain used by $\mathrm{Bu}$ and colleagues has already evolved resistance against $\beta$-sitosterol.

The key event in cell signaling transduction is the mitogen-activated protein kinase (MAPK) signaling pathway; most MAPKs are Ras dependent. Ras GTPase plays a central role in several signaling transduction pathways by regulating cell proliferation, differentiation, survival, and apoptosis [50]. Moreover, Ras GTPase is located at the beginning of the Ras signaling pathway and is involved in the activation of the Raf family and MAPK members, which inhibit apoptosis [51]. When the activity of Ras GTPase is inhibited, the activation of genes, such as Raf and $\mathrm{Bcl}-2$, is prevented but apoptosis is promoted. We found that Ras GTPase was slightly upregulated at $24 \mathrm{~h}$ posttreatment but significantly inhibited at $48 \mathrm{~h}$ posttreatment. This difference might be attributed to the fact that mites are still in the process of resisting against external compounds at an earlier time point; thus, fewer cells tend to undergo apoptosis. However, most of the energy in mites at $48 \mathrm{~h}$ posttreatment has been depleted, and more cells tend to undergo apoptosis.

\section{Conclusion}

In summary, our RNA-Seq data comprehensively show the gene expression changes in T. cinnabarinus after curcumin treatment. Differential expression was found in genes related to insecticide/acaricide targets or those involved in mite detoxification and metabolism. This study greatly expands the current understanding of the molecular response of mites towards botanical acaricide treatment. Further investigations on these differentially expressed genes will facilitate the identification of the target gene(s) of curcumin in T. cinnabarinus.

\section{Competing Interests}

The authors declare no competing interests regarding the publication of this paper.

\section{Authors' Contributions}

Wei Ding conceived and designed the experiments. Xuejiao Liu, Hong Zhou, and Ting Lai performed the experiments. Xuejiao Liu, Dousheng Wu, and Yongqiang Zhang analyzed the data. Xuejiao Liu and Wei Ding wrote the paper.

\section{Acknowledgments}

This study was financially supported by grants from the National Natural Science Foundation of China (Grants nos. 31272058 and 31572041).

\section{References}

[1] P.-Y. Mei, C. Peng, Y.-Q. Zhang, and W. Ding, "Research and application of biological acaricides," African Journal of Agricultural Research, vol. 6, no. 12, pp. 2634-2637, 2011.

[2] L. Shi, Z. Xu, G. Shen et al., "Expression characteristics of two novel cytochrome P450 genes involved in fenpropathrin resistance in Tetranychus cinnabarinus (Boisduval)," Pesticide Biochemistry and Physiology, vol. 119, no. 1, pp. 33-41, 2015.

[3] Z. Xu, L. Shi, J. Peng et al., "Analysis of the relationship between P-glycoprotein and abamectin resistance in Tetranychus cinnabarinus (Boisduval)," Pesticide Biochemistry and Physiology, vol. 129, pp. 75-82, 2016.

[4] M. Sarwar, "Management of spider mite Tetranychus cinnabarinus (Boisduval) (Tetranychidae) infestation in cotton by releasing the predatory mite Neoseiulus pseudolongispinosus (Xin, Liang and Ke) (Phytoseiidae)," Biological Control, vol. 65, no. 1, pp. 37-42, 2013.

[5] Z. Xu, W. Zhu, Y. Liu et al., "Analysis of insecticide resistancerelated genes of the carmine spider mite Tetranychus cinnabarinus based on a de novo assembled transcriptome," PLoS ONE, vol. 9, article e94779, 2014.

[6] C. Bu, B. Peng, Y. Cao et al., "Novel and selective acetylcholinesterase inhibitors for Tetranychus cinnabarinus (Acari: Tetranychidae)," Insect Biochemistry and Molecular Biology, vol. 66, pp. 129-135, 2015.

[7] P. Van Nieuwenhuyse, T. Van Leeuwen, J. Khajehali, B. Vanholme, and L. Tirry, "Mutations in the mitochondrial cytochrome b of Tetranychus urticae Koch (Acari: Tetranychidae) confer cross-resistance between bifenazate and acequinocyl," Pest Management Science, vol. 65, no. 4, pp. 404-412, 2009.

[8] T. Van Leeuwen, S. Van Pottelberge, and L. Tirry, "Comparative acaricide susceptibility and detoxifying enzyme activities in field-collected resistant and susceptible strains of Tetranychus urticae," Pest Management Science, vol. 61, no. 5, pp. 499-507, 2005.

[9] F. Guo, Z. Zhang, and Z. Zhao, "Pesticide resistance of Tetranychus cinnabarinus (Acari: Tetranychidae) in China: a review," Systematic and Applied Acarology, vol. 3, pp. 3-7, 1998.

[10] F. S. A. Mohamed, E. Hussein, Z. E. A. Darwish, S. A. A. Amer, A. B. Salama, and M. E. El-desouky, "Influence of some 
extracts from three lamiaceae plants on toxicity, repellency and some biological aspects of Tetranychus urticae Koch (Acari: Tetranychidae)," Egyptian Journal of Biological Pest Control, vol. 25, no. 1, pp. 255-261, 2015.

[11] Y. Chen and G. Dai, "Acaricidal activity of compounds from Cinnamomum camphora (L.) Presl against the carmine spider mite, Tetranychus cinnabarinus," Pest Management Science, vol. 71, no. 11, pp. 1561-1571, 2015.

[12] L.-J. Ding, W. Ding, Y.-Q. Zhang, and J.-X. Luo, "Bioguided fractionation and isolation of esculentoside $\mathrm{P}$ from Phytolacca americana L.," Industrial Crops and Products, vol. 44, pp. 534541, 2013.

[13] J. Wei, W. Ding, Y.-G. Zhao, and P. Vanichpakorn, "Acaricidal activity of Aloe vera L. leaf extracts against Tetranychus cinnabarinus (Boisduval) (Acarina: Tetranychidae)," Journal of Asia-Pacific Entomology, vol. 14, no. 3, pp. 353-356, 2011.

[14] Y. Zhang, W. Ding, and Z. Zhao, "Biological activities of curcuminoids against Tetranychus cinnabarinus Boisduval (Acari: Tetranychidae)," Acta Entomologica Sinica, vol. 50, no. 12, pp. 1304-1308, 2007.

[15] Y.-Q. Zhang, W. Ding, Z.-M. Zhao, J. Wu, and Y.-H. Fan, "Studies on Acaricidal bioactivities of Artemisia annua L. extracts against Tetranychus cinnabarinus Bois. (Acari: Tetranychidae)," Agricultural Sciences in China, vol. 7, no. 5, pp. 577-584, 2008.

[16] Y. Zhang, W. Ding, Z. Zhao, J. Wang, and H. Liao, "Research on acarcidal bioactivities of turmeric, Curcuma longa," Acta Phytophylacica Sinica, vol. 31, pp. 390-394, 2004.

[17] J. Luo, D. Wei, Y. Zhang, Z. Yang, and Y. Li, "Synthesis and acaricidal activity of curcumin pyrimidinone derivatives," Chinese Journal of Pesticide Science, vol. 13, no. 4, pp. 415-418, 2011.

[18] J. Luo, W. Ding, Y. Zhang, Z. Yang, Y. Li, and L. Ding, "Acaricidal activity of bisdemethoxycurcumin and N-methylpyrazolebisdemethoxycurcumin against Tetranychus cinnabarinus (Boisduval) and their effects on enzymes activity in the mite," Scientia Agricultura Sinica, vol. 46, pp. 2833-2844, 2013.

[19] J. Luo, W. Ding, Y. Zhang, Z. Yang, and Y. Li, "Synthesis and acaricidal activity of curcumin isoxazole and pyrazole derivatives," Chinese Journal of Pesticide Science, vol. 15, pp. 372380, 2013.

[20] L. He, L. Wang, and J. Li, "The study on the mechanism of plantinsecticide," Pesticide Science Society of Japan, vol. 34, pp. 16-19, 2013.

[21] J. Huang and W. Wu, "Review on mechanism of action and selective toxicity to new insecticides," Journal of Guizhou University, vol. 21, pp. 163-171, 2004.

[22] G. Liu, K. Furuta, H. Nakajima, F. Ozoe, and Y. Ozoe, "Competitive antagonism of insect GABA receptors by 4-substituted 5(4-piperidyl)-3-isothiazolols," Bioorganic and Medicinal Chemistry, vol. 22, no. 17, pp. 4637-4645, 2014.

[23] S. D. Buckingham, P. C. Biggin, B. M. Sattelle, L. A. Brown, and D. B. Sattelle, "Insect GABA receptors: splicing, editing, and targeting by antiparasitics and insecticides," Molecular Pharmacology, vol. 68, no. 4, pp. 942-951, 2005.

[24] J. Khajehali, T. van Leeuwen, M. Grispou et al., "Acetylcholinesterase point mutations in European strains of Tetranychus urticae (Acari: Tetranychidae) resistant to organophosphates," Pest Management Science, vol. 66, no. 2, pp. 220-228, 2010.

[25] D. H. Kwon, J. M. Clark, and S. H. Lee, "Cloning of a sodium channel gene and identification of mutations putatively associated with fenpropathrin resistance in Tetranychus urticae,"
Pesticide Biochemistry and Physiology, vol. 97, no. 2, pp. 93-100, 2010.

[26] M. Grbić, T. Van Leeuwen, R. M. Clark et al., "The genome of Tetranychus urticae reveals herbivorous pest adaptations," Nature, vol. 479, no. 7374, pp. 487-492, 2011.

[27] P. Auger, A. Migeon, E. A. Ueckermann, L. Tiedt, and M. Navajas, "Evidence for synonymy between Tetranychus urticae and Tetranychus cinnabarinus (acari, prostigmata, tetranychidae): review and new data," Acarologia, vol. 53, no. 4, pp. 383-415, 2013.

[28] S. Audic and J.-M. Claverie, "The significance of digital gene expression profiles," Genome Research, vol. 7, no. 10, pp. 986995, 1997.

[29] Y. Benjamini and D. Yekutieli, "The control of the false discovery rate in multiple testing under dependency," Annals of Statistics, vol. 29, no. 4, pp. 1165-1188, 2001.

[30] W. Sun, Y. Jin, L. He, W.-C. Lu, and M. Li, "Suitable reference gene selection for different strains and developmental stages of the carmine spider mite, Tetranychus cinnabarinus, using quantitative real-time PCR," Journal of Insect Science, vol. 10, article 208, 2010.

[31] D. Wu, W. Ding, Y. Zhang, X. Liu, and L. Yang, "Oleanolic acid induces the type III secretion system of Ralstonia solanacearum," Frontiers in Microbiology, vol. 6, article 1466, pp. 1-10, 2015.

[32] M. Zhou, C. Fan, and N. Tian, "Effects of curcumin on the gene expression profile of L-02 cells," Biomedical Reports, vol. 3, no. 4, pp. 519-526, 2015.

[33] M. A. Ortiz-Ortiz, J. M. Morán, L. M. Ruiz-Mesa et al., "Curcumin exposure induces expression of the Parkinson's disease-associated leucine-rich repeat kinase 2 (LRRK2) in rat mesencephalic cells," Neuroscience Letters, vol. 468, no. 2, pp. 120-124, 2010.

[34] Y.-F. Liao, H.-C. Hung, T.-C. Hour et al., "Curcumin induces apoptosis through an ornithine decarboxylase-dependent pathway in human promyelocytic leukemia HL-60 cells," Life Sciences, vol. 82, no. 7-8, pp. 367-375, 2008.

[35] S. S. Han, S. T. Chung, D. A. Robertson, D. Ranjan, and S. Bondada, "Curcumin causes the growth arrest and apoptosis of B cell lymphoma by downregulation of egr-1, c-myc, bcl-XL, NF-kappa B, and p53," Clinical Immunology, vol. 93, no. 2, pp. 152-161, 1999.

[36] L. R. Chaudhary and K. A. Hruska, "Inhibition of cell survival signal protein kinase B/Akt by curcumin in human prostate cancer cells," Journal of Cellular Biochemistry, vol. 89, no. 1, pp. $1-5,2003$.

[37] J.-H. Woo, Y.-H. Kim, Y.-J. Choi et al., "Molecular mechanisms of curcumin-induced cytotoxicity: Induction of apoptosis through generation of reactive oxygen species, downregulation of $\mathrm{Bcl}-\mathrm{XL}$ and IAP, the release of cytochrome $\mathrm{c}$ and inhibition of Akt," Carcinogenesis, vol. 24, no. 7, pp. 1199-1208, 2003.

[38] J.-Y. Liu, S.-J. Lin, and J.-K. Lin, "Inhibitory effects of curcumin on protein kinase $\mathrm{C}$ activity induced by $12-\mathrm{O}$-tetradecanoylphorbol-13-acetate in MH 3T3 cells," Carcinogenesis, vol. 14, no. 5, pp. 857-861, 1993.

[39] C. Li, "The toxic symptoms and penetration effect of integument of Tetranychus cinnabarinus after treatment with curcumin," 2015.

[40] W. Y. Cheung, "Calmodulin plays a pivotal role in cellular regulation," Science, vol. 207, no. 4426, pp. 19-27, 1980. 
[41] Y. Shirasaki, Y. Kanazawa, Y. Morishima, and M. Makino, "Involvement of calmodulin in neuronal cell death," Brain Research, vol. 1083, no. 1, pp. 189-195, 2006.

[42] C. Montecucco, J. M. Gutiérrez, and B. Lomonte, "Cellular pathology induced by snake venom phospholipase A2 myotoxins and neurotoxins: common aspects of their mechanisms of action," Cellular and Molecular Life Sciences, vol. 65, no. 18, pp. 2897-2912, 2008.

[43] J. Šribar, J. Oberčkal, and I. Križaj, "Understanding the molecular mechanism underlying the presynaptic toxicity of secreted phospholipases A2: an update," Toxicon, vol. 89, pp. 9-16, 2014.

[44] J. I. Yeh, U. Chinte, and S. Du, "Structure of glycerol-3phosphate dehydrogenase, an essential monotopic membrane enzyme involved in respiration and metabolism," Proceedings of the National Academy of Sciences of the United States of America, vol. 105, no. 9, pp. 3280-3285, 2008.

[45] D. S. Buss and A. Callaghan, "Interaction of pesticides with pglycoprotein and other ABC proteins: a survey of the possible importance to insecticide, herbicide and fungicide resistance," Pesticide Biochemistry and Physiology, vol. 90, no. 3, pp. 141-153, 2008.

[46] W. Dermauw and T. Van Leeuwen, "The ABC gene family in arthropods: comparative genomics and role ininsecticide transport and resistance," Insect Biochemistry and Molecular Biology, vol. 45, no. 1, pp. 89-110, 2014.

[47] B. F. Ardelli, "Transport proteins of the ABC systems superfamily and their role in drug action and resistance in nematodes," Parasitology International, vol. 62, no. 6, pp. 639-646, 2013.

[48] G. Broehan, T. Kroeger, M. Lorenzen, and H. Merzendorfer, "Functional analysis of the ATP-binding cassette (ABC) transporter gene family of Tribolium castaneum," BMC Genomics, vol. 14, article 6, 2013.

[49] C. Bu, J. Li, X.-Q. Wang et al., "Transcriptome analysis of the carmine spider mite, Tetranychus cinnabarinus (Boisduval, 1867) (Acari: Tetranychidae), and its response to $\beta$-sitosterol," BioMed Research International, vol. 2015, Article ID 794718, 12 pages, 2015.

[50] R. Knihtila, G. Holzapfel, K. Weiss, F. Meilleur, and C. Mattos, "Neutron crystal structure of RAS GTPase puts in question the protonation state of the GTP $\gamma$-phosphate," The Journal of Biological Chemistry, vol. 290, no. 52, pp. 31025-31036, 2015.

[51] I. F. Benter, J. S. Juggi, I. Khan, and S. Akhtar, "Inhibition of Ras-GTPase, but not tyrosine kinases or $\mathrm{Ca}^{2+} /$ calmodulindependent protein kinase II, improves recovery of cardiac function in the globally ischemic heart," Molecular and Cellular Biochemistry, vol. 259, no. 1-2, pp. 35-42, 2004. 

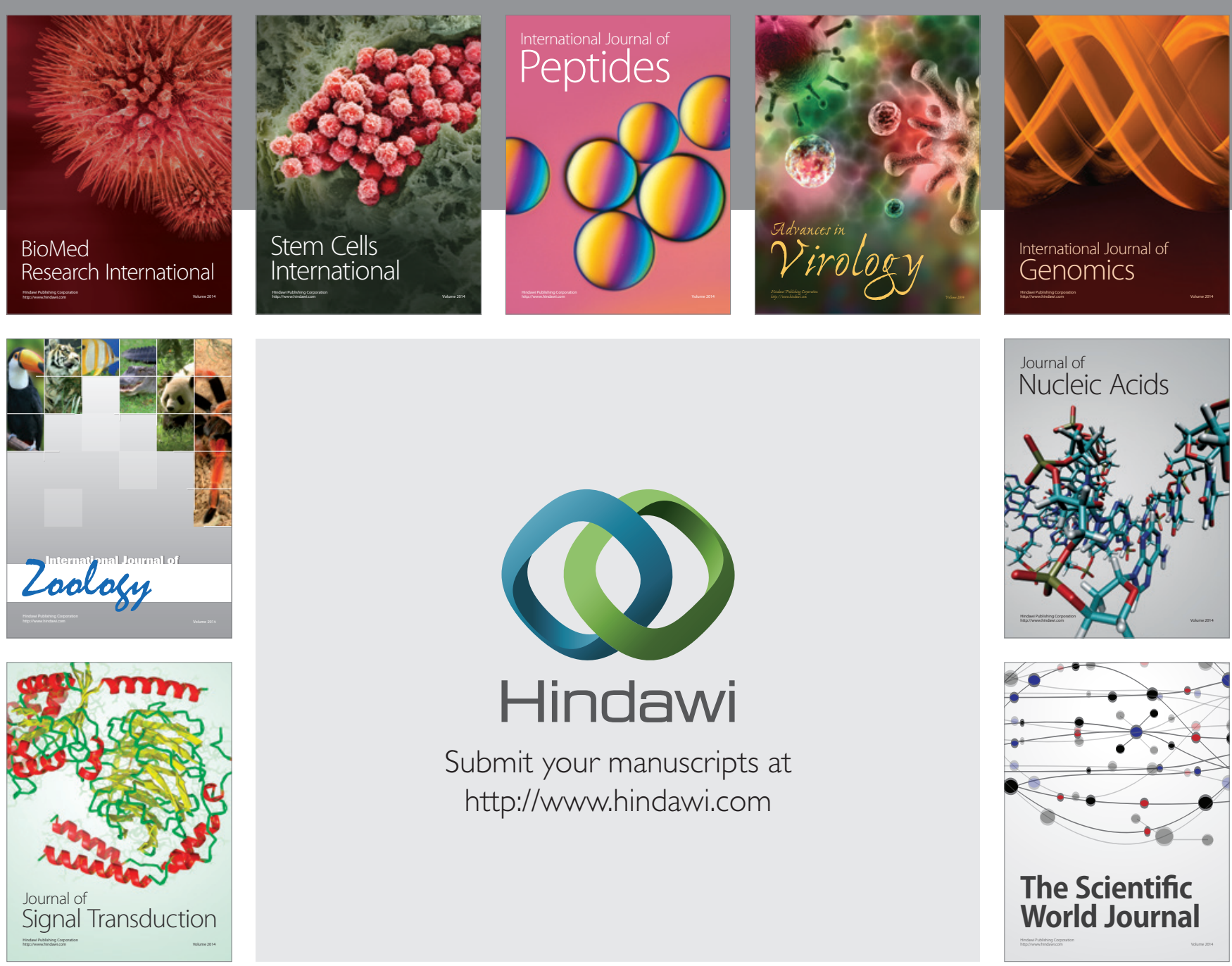

Submit your manuscripts at

http://www.hindawi.com
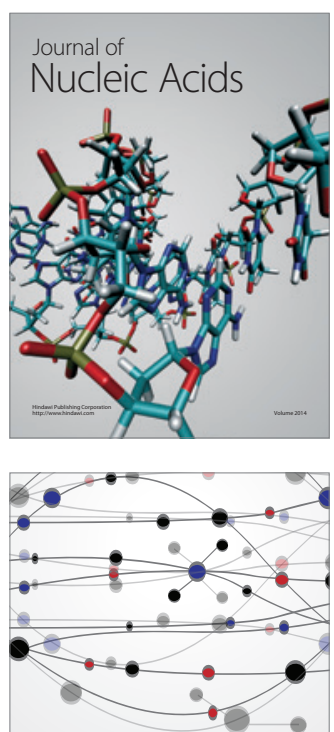

The Scientific World Journal
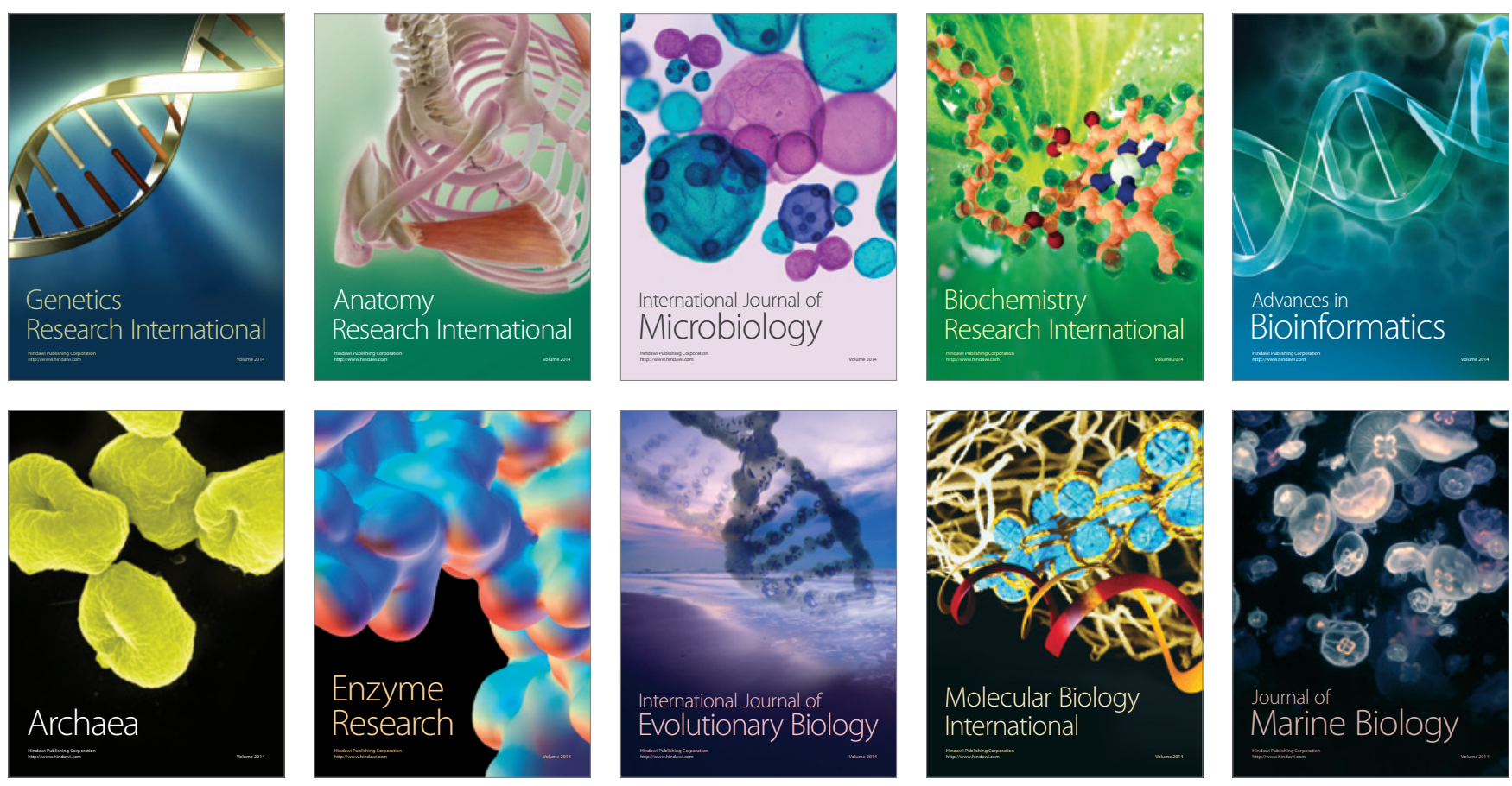